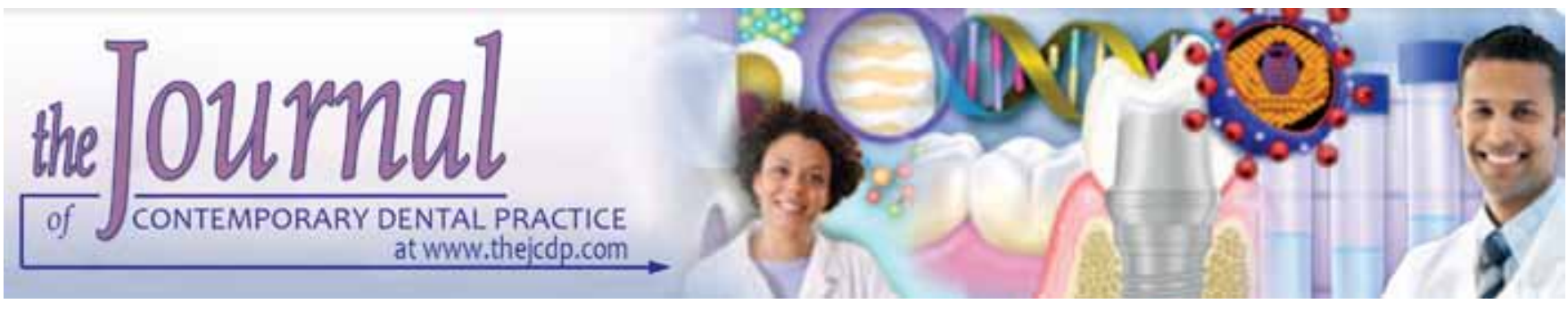

\title{
Objective Evaluation of Cervical Vertebral Bone Age- Its Reliability in Comparison with Hand-Wrist Bone Age: By TW3 Method
}

\author{
CMS Krishna Prasad, Vamsi Nilay Reddy, Gojja Sreedevi, Swaroopa Rani Ponnada \\ K Padma Priya, B Raveendra Naik
}

\section{ABSTRACT}

Aim: The aim of this study was to establish the validity of a new method for evaluating skeletal maturation by assessing the 3rd and 4th cervical vertebrae seen in the cephalometric radiograph.

Materials and methods: This study consisted of a sample of 50 patients in the age group of 8 to 14 years of age. Chronologically, they were divided into six groups, based on the age consisting of a minimum of six to a maximum of 10 subjects. All the patients included in the study were females. The selected subjects were clinically examined and then age and date of birth of the patient in years and months was noted. Then lateral cephalograms and hand-wrist radiographs of the patient were taken on the same day with good clarity and contrast.

Results: The results suggested that cervical vertebral bone age on cephalometric radiographs calculated with this method is as reliable at estimating bone age as is the Tanner-Whitehouse 3 (TW3) method on hand-wrist radiographs. By determining the cervical vertebral bone age, skeletal maturity can be evaluated in a detailed and objective manner with cephalometric radiographs.

Conclusion: The ability to accurately appraise skeletal maturity from cervical vertebral maturation, without the need for additional radiographs, has the potential to improve orthodontic diagnostic and therapeutic decisions. The technique's simplicity and ease of use should encourage this method as a first level diagnostic tool to assess skeletal maturation.

Clinical significance: This study revealed that the timing and sequence of ossification of the bones in hand and wrist and cervical vertebrae were able to relate the skeletal development of the various skeletal maturity indicators to a child's development. This method provided a mean with which one can determine the skeletal maturity of a person and thereby determine whether the possibility of potential growth existed.

Keywords: Skeletal maturation, Lateral, Cephalograms, Handwrist radiographs, Cervical vertebral bone, Tanner-Whitehouse 3 method.

How to cite this article: Prasad CMSK, Reddy VN, Sreedevi G, Ponnada SR, Priya KP, Naik BR. Objective Evaluation of Cervical Vertebral Bone Age-Its Reliability in Comparison with Hand-Wrist Bone Age: By TW3 Method. J Contemp Dent Pract 2013;14(5):806-813.

\section{Source of support: Nil}

Conflict of interest: None declared

\section{INTRODUCTION}

The evaluation of craniofacial growth is an essential part of diagnosis and treatment planning in orthodontics. Assessment of growth and development is a prime concern in caring for the growing child and adolescent, especially when growth modification is needed. Biological age, skeletal age, bone age and skeletal maturation are nearly synonymous terms used to describe the stage of maturation of a person. ${ }^{1}$ Sexual maturation characteristics, chronologic age, dental development, height, weight and skeletal development are some of the more common means that have been used to identify stages of growth. Because of individual variations in timing, duration and velocity of growth, skeletal age assessment is essential in formulating viable orthodontic treatment plans. ${ }^{2}$ Skeletal maturation refers to the degree of development of ossification in bone.

The early prevention and interception of dental deformities depend upon an accurate interpretation of the inherent facioskeletal pattern and the overall growth and development. ${ }^{3}$ During growth, every bone goes through a series of changes that can be seen radiologically. The sequence of changes is relatively consistent for a given bone in every person. The timing of the changes varies because each person has his or her own biologic clock. ${ }^{4}$

The idea of accurately determining the skeletal age of patients was to coordinate this information with orthodontic treatment so as to maximize the therapeutic effect. Unfortunately, a low correlation has been found between general skeletal maturity and facial growth as measured by common parameters. ${ }^{5}$ 
The standard method of evaluating skeletal maturity has been to use a hand-wrist radiograph. Assessment of skeletal maturation using hand-wrist radiograph as an index based upon time and sequence of appearance of carpal bones and certain ossification events has been reported by many investigators. ${ }^{6-8}$ To avoid taking an additional radiograph, however, some researchers have sought to relate maturation with dental and skeletal features other than the bones in the hand and wrist. ${ }^{9}$

The use of cervical vertebrae to determine skeletal maturity is not new. Lamparski ${ }^{10}$ in 1972 found that cervical vertebrae, as seen on routine lateral cephalograms were as statistically and clinically reliable in assessing skeletal age as the hand-wrist technique. ${ }^{9}$ He published an atlas that simulated the morphological changes in cervical vertebral bodies in puberty and used these changes to evaluate skeletal maturation.

Other researchers have confirmed the validity of Lamparski's ${ }^{10}$ method of evaluating the skeletal maturity of orthodontic patients. ${ }^{11,12}$ Garcia-Fernandez et $\mathrm{al}^{9}$ reported a high correlation between cervical vertebral maturation using the atlas and skeletal maturation of the hand wrist. Their techniques did not require hand-wrist radiographs and could be used to roughly evaluate pubertal growth based on cephalometric radiographs. However, these techniques could not be used to evaluate growth in a detailed and objective manner, because they used an atlas.

\section{AIMS AND OBJECTIVES}

The purpose of this study was to establish the validity of a new method given by T Mito and K Sato ${ }^{13}$ in 2002 for objectively evaluating skeletal maturation by assessing the 3 rd and 4rth cervical vertebrae seen in the cephalometric radiographs and the validity was established by comparing the cervical vertebral bone age determined by using the formula given by $\mathrm{T}$ Mito and K Sato with bone age determined with hand-wrist radiographs, using the TannerWhitehouse 3 (TW3) method. ${ }^{14}$

\section{MATERIALS AND METHODS (FIGS 1 TO 10)}

This study consisted of a sample of 50 patients in the age group of 8 to 14 years of age. Chronologically, there were divided into six groups, based on the age consisting of a minimum of six to a maximum of 10 subjects. All the patients included in the study were females.

Materials and methods required:

1. Lateral cephalograms $-8 \times 10$ inches

2. Hand-wrist radiographs $-8 \times 10$ inches

3. $50 \mu$ thick Matt acetate tracing paper

4. Lead pencil $2 \mathrm{~B}$

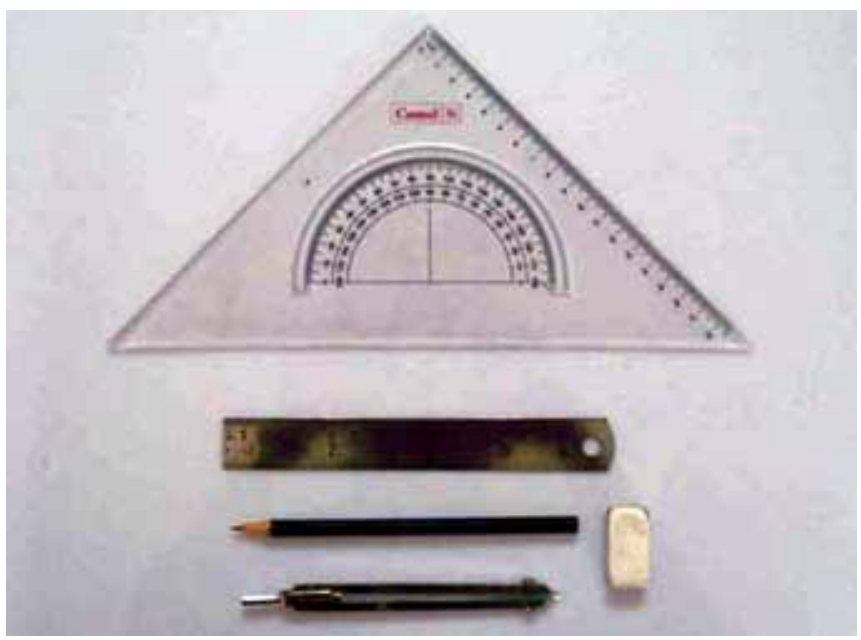

Fig. 1: The armamentarium used

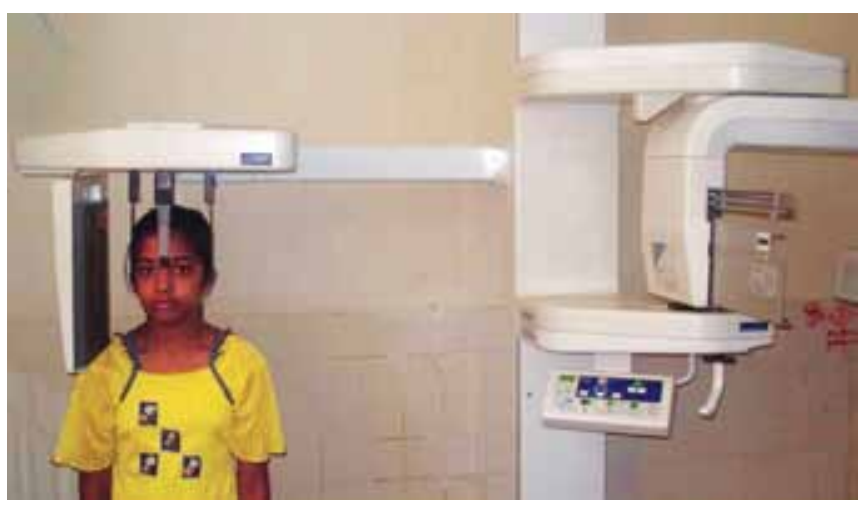

Fig. 2: Patient positioning for lateral cephalogram

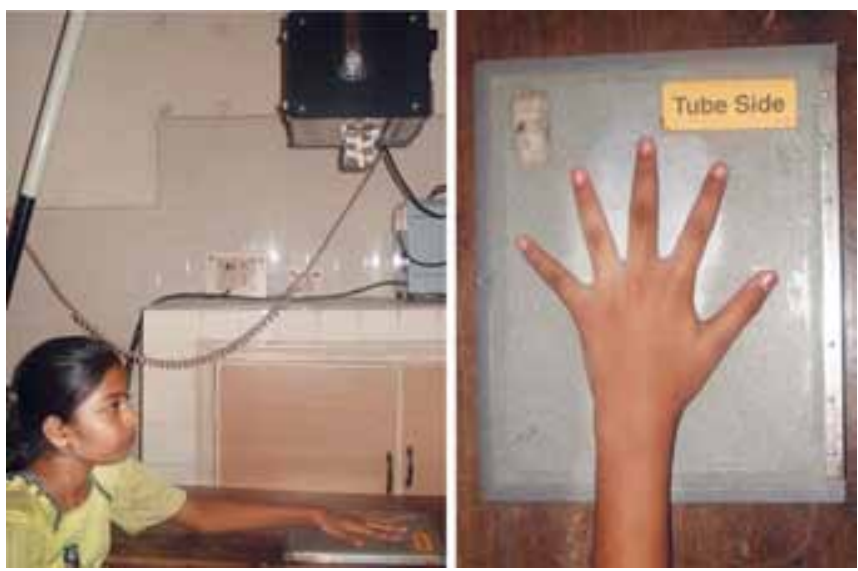

Fig. 3: Hand-wrist radiographic machine and patient positioning for hand-wrist radiograph

5. Radiographic view box

6. Metal scale

7. Calipers

The selected subjects were clinically examined and then age and date of birth of the patient in years and months was noted. Then lateral cephalograms and hand-wrist radiographs of the patient were taken on the same day with good clarity and contrast and tracing technique was followed. To eliminate error all the tracings were done by a single operator. 


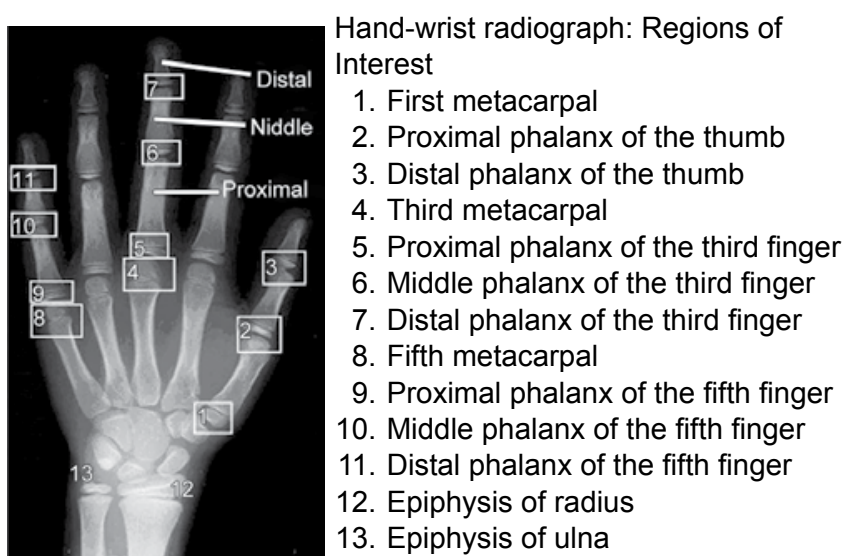

Fig. 4: Hand-wrist radiograph regions of interest
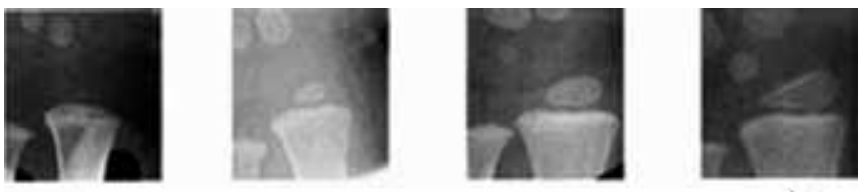

B

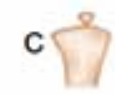

D
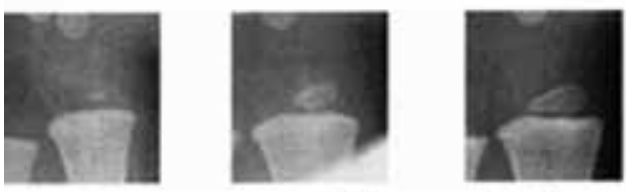

Radius-stage $\mathrm{B}$ to $\mathrm{E}$
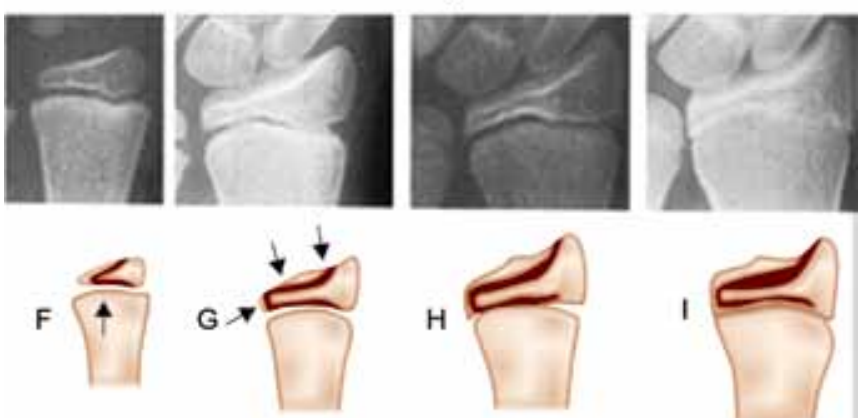

$\mathrm{H}$
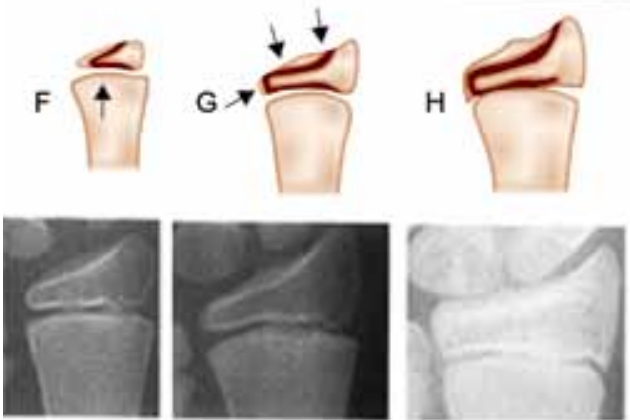

Radius-stage $\mathrm{F}$ to $\mathrm{I}$

Fig. 5: Stages of skeletal maturity-TW3 method for radius (stage B-stage $\mathrm{E}$ and stage F-stage I)

\section{Skeletal Age Assessment}

\section{Hand-Wrist Bone Age Determination}

In this study, the skeletal maturity indicators of hand-wrist were evaluated using the TW3 method. ${ }^{14}$ Bone age in this study was calculated using the radius, ulna and short bones (RUS) scores of the TW3 method. In the RUS scoring method 13 bones are examined. They are rated in the following order: radius, ulna, metacarpals I, III, V; proximal
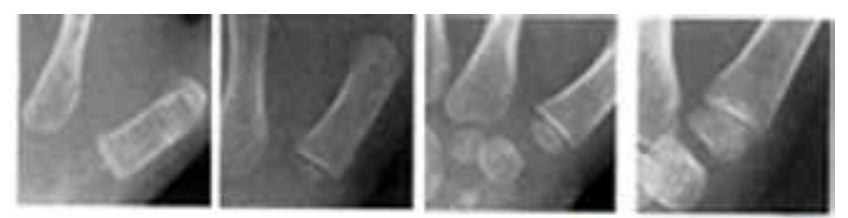

B
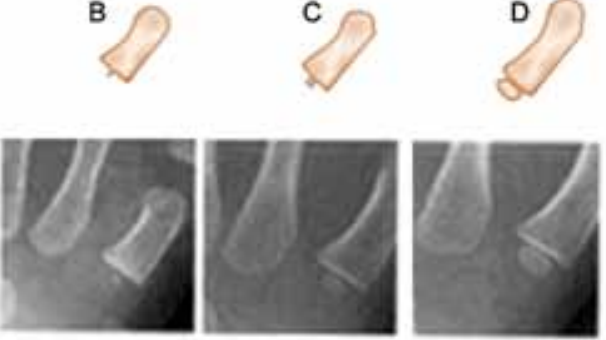

Stage $B$ to $E$
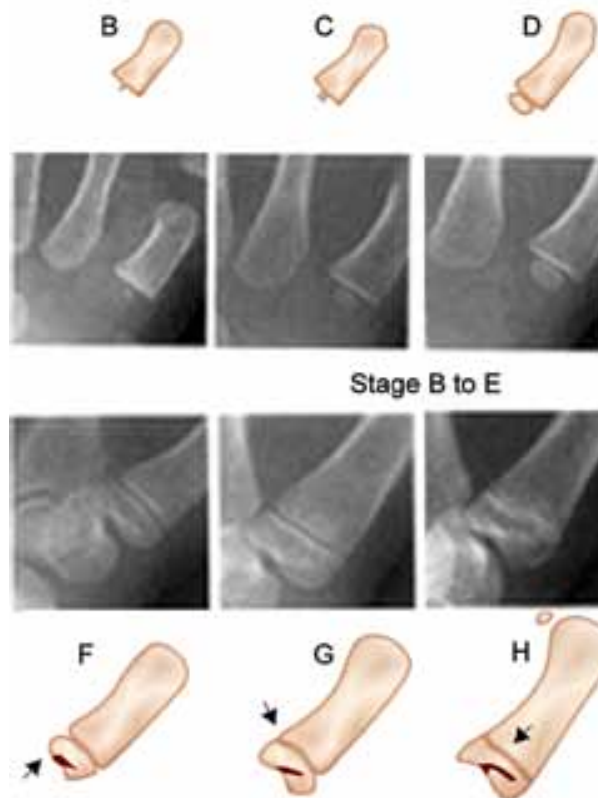

E
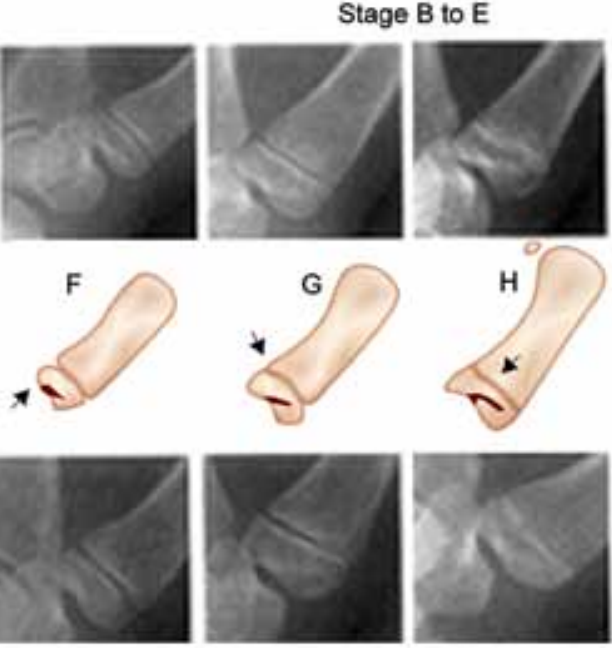

Stage $F$ to $I$

Fig. 6: Stages of skeletal maturity-TW3 method for first metacarpal (stage B-stage $\mathrm{E}$ and stage $\mathrm{F}$-stage I)

phalanges I, III, V; middle phalanges III, V; distal phalanges I, III, V.

\section{Cervical Vertebral Bone Age Determination}

\section{Methods}

On lateral cephalometric radiographs the third and fourth cervical vertebrae were traced by a pencil and the following parameters were measured with a calipers: anterior vertebral body height $(\mathrm{AH})$, posterior vertebral body height $(\mathrm{PH})$, and anteroposterior body length (AP) on the third and fourth cervical vertebrae.

Now, using the formula given by Mito et al cervical vertebral bone age was calculated.

Cervical vertebral bone age $=-0.20+6.20 \times \mathrm{AH}_{3} / \mathrm{AP}_{3}+$ $5.90 \times \mathrm{AH}_{4} / \mathrm{AP}_{4}+4.74 \times \mathrm{AH}_{4} / \mathrm{PH}_{4}$

After calculating the skeletal maturity by both the methods the correlation and difference between cervical vertebral bone age and bone age by hand-wrist method were determined, as were those between cervical vertebral bone age and chronological age. 


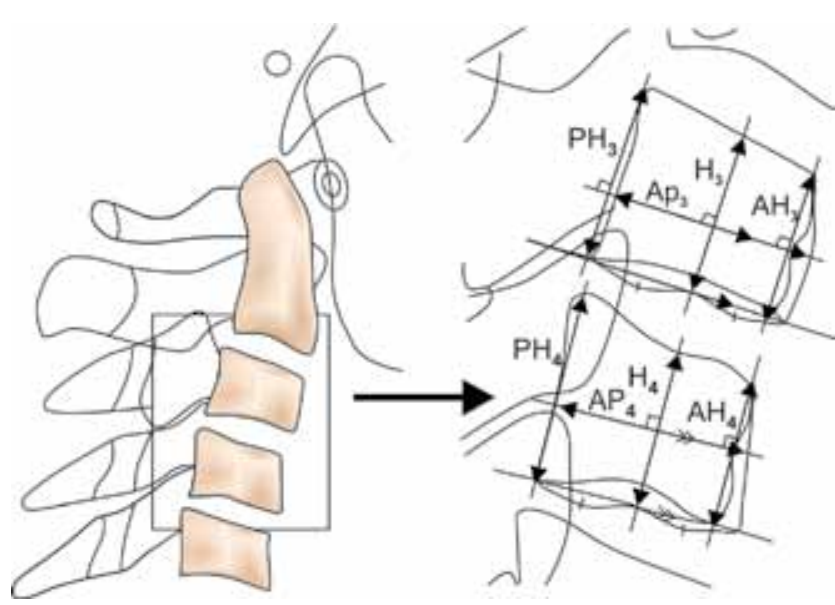

Lower lines are tangent to front and back of lower parts of cervical vertebral bodies $\left(\mathrm{AH}_{3}, \mathrm{AH}_{4}\right.$ : distance from top of front part to tangent of lower part; $\mathrm{H}_{3}, \mathrm{H}_{4}$ : distance from top of middle part to tangent of lower part; $\mathrm{PH}_{3}, \mathrm{PH}_{4}$ : distance from top of back part to tangent of lower part; $\mathrm{AP}_{3}, \mathrm{AP}_{4}$ : anteroposterior distance at middle of cervical vertebral body).

Fig. 7: Cervical bone age estimation by Mito et al method

\section{RESULTS}

\section{Statistical Analysis}

A paired t-test was used to determine if there was a significant difference in the correlation coefficients between cervical vertebral bone age and bone age, and between cervical vertebral bone age and chronological age.

As shown in Tables 1 to 5 and represented in Graphs 1 and 2, the correlation coefficient between cervical vertebral bone age and bone age by the TW3 method $(r=0.915)$ was significantly $(\mathrm{p}=0.000)$ higher than that between cervical vertebral bone age and chronological age $(r=0.797)$ and also higher than that between bone age and chronological age $(r=0.844)$.

However, the average difference in years between cervical vertebral bone age and bone age $(0.170 \pm 1.08$ years) and that between cervical vertebral bone age and chronological age $(0.097 \pm 0.793$ years $)$ was not statistically
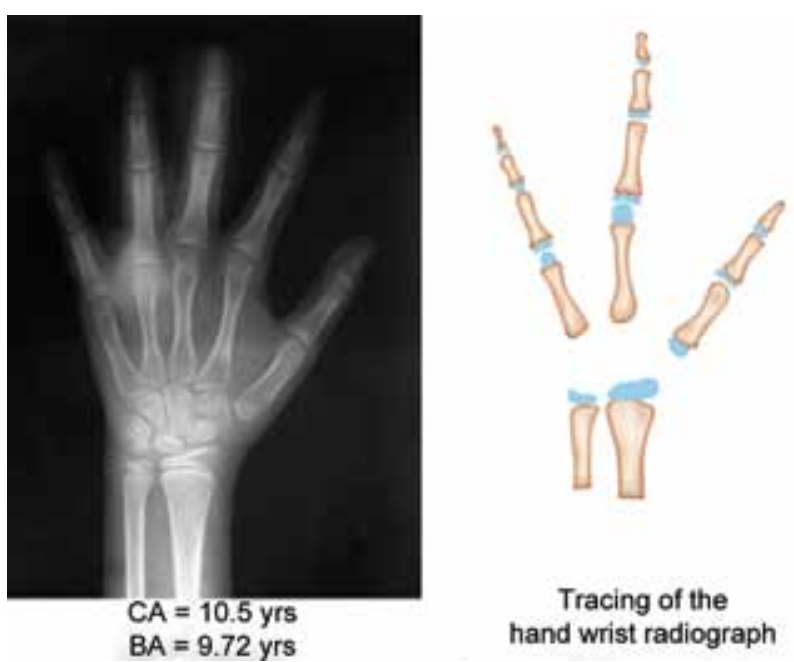

Fig. 9: Hand-wrist radiograph of a 10-year-old patient and tracing of the hand-wrist radiograph
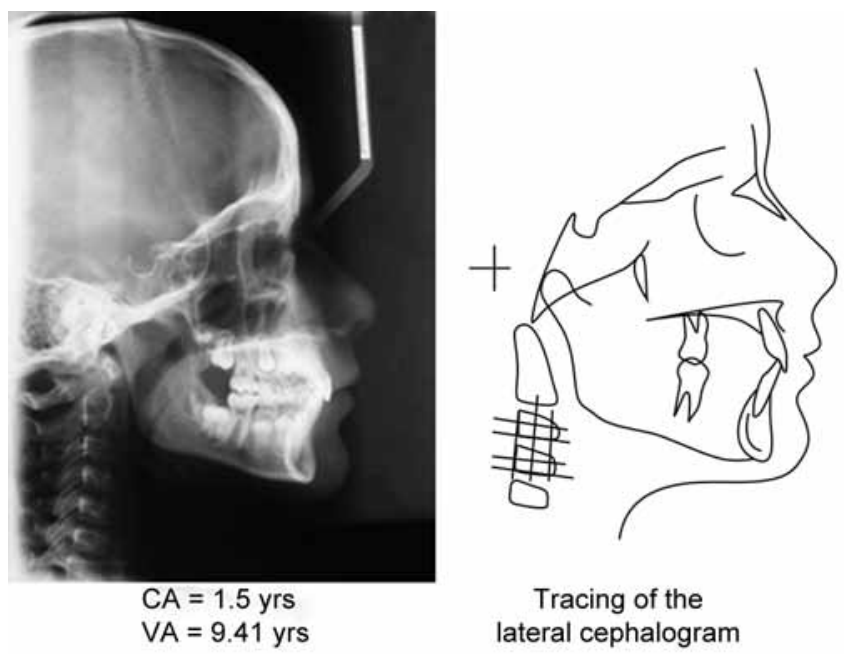

Tracing of the lateral cephalogram

Fig. 10: Lateral cephalogram of the same 10-year-old patient and tracing of the cervical vertebrae in the lateral cephalogram

significant. The results suggest that the sample size needed to be increased to form a conclusion regarding the difference (in absolute values) between the parameters.

Thus, the results and the statistical analysis suggest that the relation between the cervical vertebral bone age

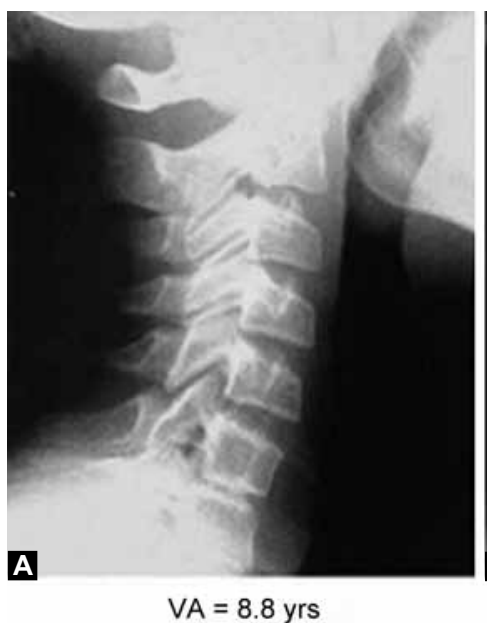

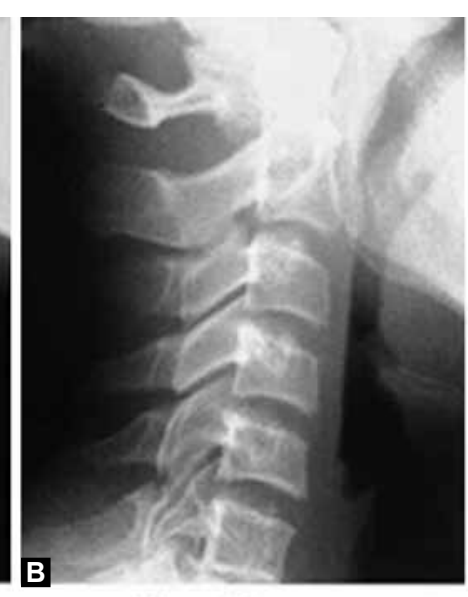

$\mathrm{VA}=11.8 \mathrm{yrs}$

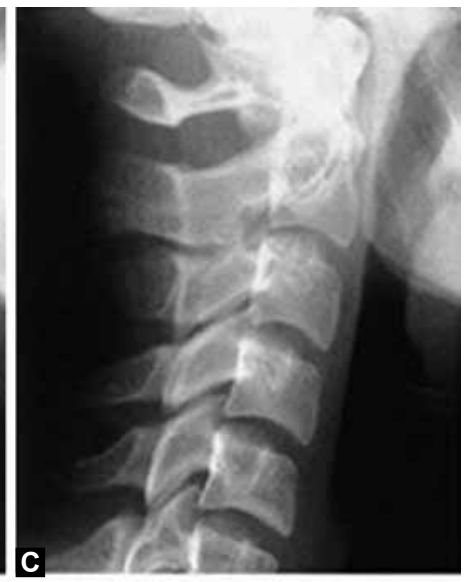

$\mathrm{VA}=13.4 \mathrm{yrs}$

Figs 8 A to C: Radiographs showing stages of cervical vertebral maturation 
estimated with the Mito et al method and bone age is highly significant and higher than the correlations between the other parameters in the study.
The calculated cervical vertebral bone age and bone age by the hand-wrist radiographs have been showed here in the Table 1.

\begin{tabular}{|c|c|c|c|c|c|}
\hline S. no & $C A$ & $B A$ & $V A$ & $C A-V A$ & $B A-V A$ \\
\hline 1. & 13.8 & 14.45 & 14.28 & -0.48 & 0.17 \\
\hline 2. & 13 & 12.2 & 11.338 & 1.662 & 0.862 \\
\hline 3. & 9.667 & 10.1 & 9.86 & -0.193 & 0.24 \\
\hline 4. & 11.5 & 12.7 & 10.61 & 0.89 & 2.09 \\
\hline 5. & 9.25 & 9.5 & 9.197 & 0.053 & 0.303 \\
\hline 6. & 8.834 & 9.45 & 9.645 & -0.811 & -0.195 \\
\hline 7. & 8.83 & 8.2 & 9.4 & -0.57 & -1.2 \\
\hline 8. & 11.25 & 9.95 & 9.449 & 1.801 & 0.501 \\
\hline 9. & 9.416 & 10 & 10.235 & -0.819 & -0.235 \\
\hline 10. & 10.583 & 10.6 & 9.55 & 1.033 & 1.05 \\
\hline 11. & 9.25 & 9.65 & 8.783 & 0.467 & 0.867 \\
\hline 12. & 9.5 & 9.1 & 9.841 & -0.341 & -0.741 \\
\hline 13. & 8.5 & 9.65 & 10.107 & -1.607 & -0.457 \\
\hline 14. & 10.667 & 11.45 & 9.335 & 1.332 & 2.115 \\
\hline 15. & 13.416 & 14.1 & 13.362 & 0.054 & 0.738 \\
\hline 16. & 8.083 & 8 & 8.234 & -0.151 & -0.234 \\
\hline 17. & 11.916 & 11.2 & 11.357 & 0.559 & -0.157 \\
\hline 18. & 12.667 & 12.3 & 12.712 & -0.045 & -0.412 \\
\hline 19. & 12.667 & 12.2 & 11.553 & 1.114 & 0.647 \\
\hline 20. & 10.167 & 10.35 & 10.756 & -0.589 & -0.406 \\
\hline 21. & 12.916 & 12.2 & 11.49 & 1.426 & 0.71 \\
\hline 22. & 13.083 & 13.05 & 13.957 & -0.874 & -0.907 \\
\hline 23. & 12.5 & 12.2 & 11.793 & 0.707 & 0.407 \\
\hline 24. & 9.667 & 8.45 & 8.973 & 0.694 & -0.523 \\
\hline 25. & 8.667 & 7.83 & 8.336 & 0.331 & -0.506 \\
\hline 26. & 12.667 & 12.2 & 12.305 & 0.362 & -0.105 \\
\hline 27. & 12.25 & 11.1 & 10.61 & 1.64 & 0.49 \\
\hline 28. & 12.334 & 11.25 & 10.597 & 1.737 & 0.653 \\
\hline 29. & 11.25 & 13.55 & 12.452 & -1.202 & 1.098 \\
\hline 30. & 11.416 & 10.6 & 10.93 & 0.486 & -0.33 \\
\hline 31. & 8.583 & 6.35 & 7.8 & 0.783 & -1.45 \\
\hline 32. & 9.416 & 12.15 & 12.062 & -2.646 & 0.088 \\
\hline 33. & 13.75 & 15 & 14.404 & -0.654 & 0.596 \\
\hline 34. & 10.583 & 10.45 & 9.712 & 0.871 & 0.738 \\
\hline 35. & 10.083 & 10.3 & 10.204 & -0.121 & 0.096 \\
\hline 36. & 12.667 & 12 & 12.447 & 0.22 & -0.447 \\
\hline 37. & 12.5 & 12.2 & 11.556 & 0.944 & 0.644 \\
\hline 38. & 12.834 & 12.2 & 11.827 & 1.007 & 0.373 \\
\hline 39. & 9.083 & 11.8 & 11.941 & -2.858 & -0.141 \\
\hline 40. & 12 & 11.9 & 11.098 & 0.902 & 0.802 \\
\hline 41. & 11 & 12.2 & 12.672 & -1.672 & -0.472 \\
\hline 42. & 8 & 8.35 & 8.849 & -0.849 & -0.499 \\
\hline 43. & 10.167 & 8.65 & 8.836 & 1.331 & -0.186 \\
\hline 44. & 11.75 & 11.7 & 11.514 & 0.236 & 0.186 \\
\hline 45. & 13.583 & 12.2 & 11.94 & 1.643 & 0.26 \\
\hline 46. & 8 & 6.15 & 8.534 & -0.534 & -2.384 \\
\hline 47. & 8.667 & 9 & 9.375 & -0.708 & -0.375 \\
\hline 48. & 12.5 & 12.25 & 12.695 & -0.195 & -0.445 \\
\hline 49. & 11 & 9.95 & 9.794 & 1.206 & 0.156 \\
\hline 50. & 10.834 & 10.7 & 9.894 & 0.97 & 0.806 \\
\hline
\end{tabular}

CA: Chronological age; A: Hand-wrist bone age; VA: Vertebral age; CA-VA: Absolute difference between chronological age and vertebral age; BA-VA: Absolute difference between hand-wrist bone age and vertebral age 


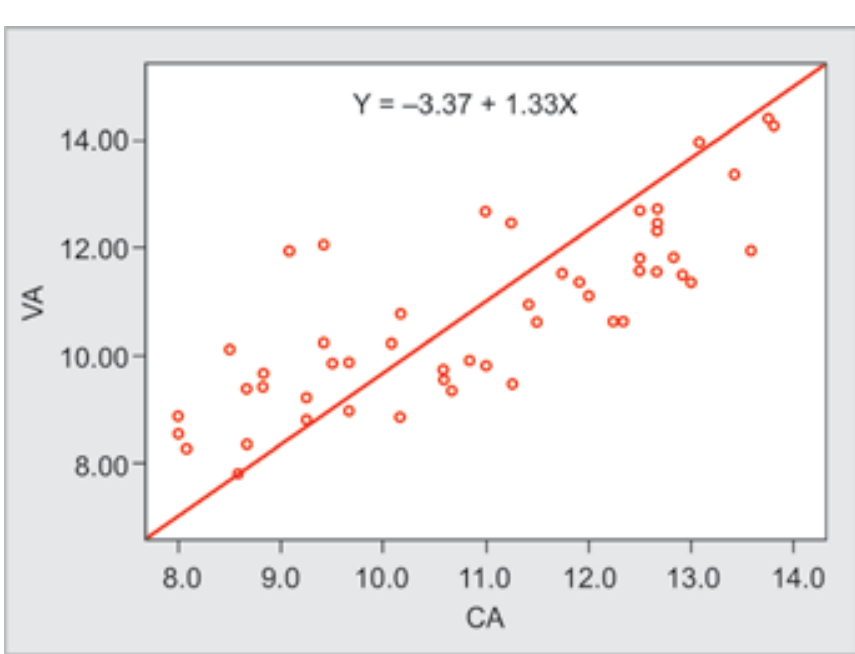

Graph 1: Scattergram of cervical vertebral bone age (VA) and chronological age (CA)

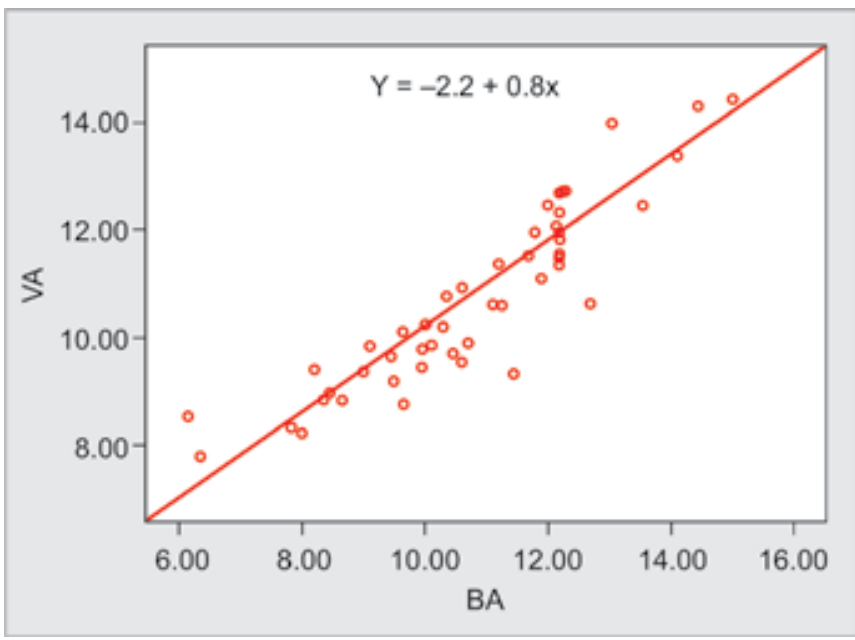

Graph 2: Scattergram of cervical vertebral bone age (VA) and bone age (BA)

\begin{tabular}{llllll}
\multicolumn{6}{c}{ Table 2: Paired samples statistics } \\
\hline & & Mean & $N$ & $\begin{array}{l}\text { Std. } \\
\text { deviation }\end{array}$ & $\begin{array}{l}\text { Std. error } \\
\text { mean }\end{array}$ \\
\hline Pair 1 & CA & 10.934 & 50 & 1.7464 & 0.2470 \\
& VA & 10.7640 & 50 & 1.63174 & 0.23076 \\
Pair 2 & BA & 10.8616 & 50 & 1.93554 & 0.27373 \\
& VA & 10.7640 & 50 & 1.63174 & 0.23076 \\
\hline
\end{tabular}

\begin{tabular}{lllll}
\multicolumn{5}{c}{ Table 3: Paired samples correlations } \\
\hline & & $N$ & Correlation & Significance \\
\hline Pair 1 & CA-VA & 50 & 0.797 & 0.000 \\
Pair 2 & BA-VA & 50 & 0.915 & 0.000 \\
\hline
\end{tabular}

\section{DISCUSSION}

The growth factor is a critical variable in orthodontic treatment. A treatment plan can vary from orthognathic surgery to extraction of teeth to nonextraction of teeth, depending on the growth factor. ${ }^{2}$
Genetic and racial diversity and other environmental influences have a marked effect on the rate of development of the prepubertal and pubertal growth of the child. Skeletal maturity among all is the most commonly used index in routine clinical work and is closely related to the somatic and sexual maturity. Every person matures on an individual schedule and it is here that the value of skeletal age assessment becomes apparent.

A more accurate assessment of the physiological development can be made by using radiographic examination of the calcified structures of the hand-wrist. ${ }^{15}$

The maturational changes of the cervical vertebrae as seen on the lateral cephalogram are clinically reliable in assessing skeletal age. Knowledge of these stages of maturation that a child has attained helps in evaluating his/her progression through developmental status. The information bears great clinical importance in identifying the optimal time for prompt orthodontic management of the child. ${ }^{16}$

The use of cervical vertebrae to determine skeletal maturity is not new. ${ }^{9}$ Lamparski ${ }^{10}$ was the first to use cervical vertebrae as indicators for skeletal maturation. He published an atlas that simulated the morphological changes in the cervical vertebral bodies in puberty and used these changes to evaluate skeletal maturation.

Cervical vertebrae $\mathrm{C} 2$ to $\mathrm{C} 6$ were used in this study. Since, these vertebrae were already recorded in the routine lateral cephalogram, there was no need for additional radiographic exposures. Use of thyroid collar blocks 5 th and 6 th cervical vertebrae out of radiograph images. Almost all previous evaluations in puberty with cervical vertebrae on cephalometric radiographs either used or referred to the atlas reported by Lamparski. ${ }^{10}$

The present study was undertaken with the aim to check the validity of this new method proposed by Mito et al for objectively evaluating skeletal maturation on a cephalometric radiograph. They measured vertebral bodies of the third and fourth cervical vertebrae and omitted other cervical vertebrae for various reasons: the first cervical vertebra (atlas) does not show the body, the second cervical vertebra (axis), shows very little morphological change and is difficult to measure, and the fifth cervical vertebra might not appear clearly on cephalometric radiographs. Ratios were used to calculate cervical vertebral bone age because this considers only the shape of cervical vertebrae and discounts their size.

To confirm the validity of this formula the correlation and difference between cervical bone age from this method and bone age determined by the hand-wrist radiographs using the RUS scores of the TW3 method was calculated 


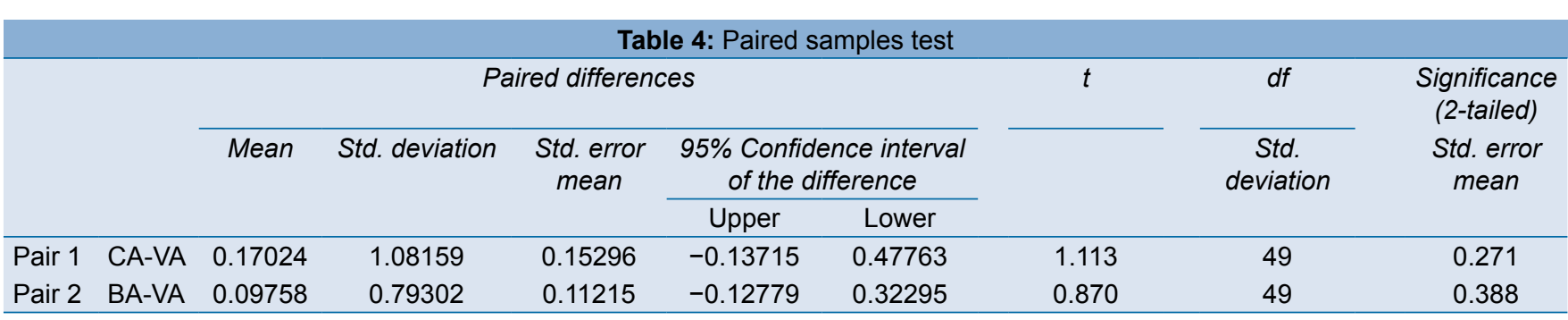

\begin{tabular}{|c|c|c|c|c|}
\hline \multicolumn{5}{|c|}{ Table 5: Correlations } \\
\hline & & $C A$ & $B A$ & $V A$ \\
\hline \multirow[t]{3}{*}{ CA } & Pearson correlation & 1 & $0.844^{\star *}$ & $0.797^{\star *}$ \\
\hline & significance (2-tailed) & & 0.000 & 0.000 \\
\hline & $\mathrm{N}$ & 50 & 50 & 50 \\
\hline \multirow[t]{3}{*}{ BA } & Pearson correlation & $0.844^{* *}$ & 1 & $0.915^{\star *}$ \\
\hline & significance (2-tailed) & 0.000 & & 0.000 \\
\hline & $\mathrm{N}$ & 50 & 50 & 50 \\
\hline \multirow[t]{3}{*}{ VA } & Pearson correlation & $0.797^{* *}$ & $0.915^{\star *}$ & 1 \\
\hline & significance (2-tailed) & 0.000 & 0.000 & \\
\hline & $\mathrm{N}$ & 50 & 50 & 50 \\
\hline
\end{tabular}

${ }^{* *}$ Correlation is significant at the 0.01 level (2-tailed)

and also between cervical vertebral bone age and the chronological age. Our study showed that the correlation coefficient between cervical vertebral bone age and bone age by the TW3 method was significantly higher than that between cervical vertebral bone age and chronological age and also that between bone age and chronological age. Thus, indicating that cervical vertebral bone age more closely approximates bone age by the hand-wrist radiographs by the TW3 method which is considered to be the most reliable method for measuring the degree of maturity.

This study revealed that the timing and sequence of ossification of the bones in hand and wrist and cervical vertebrae were able to relate the skeletal development of the various skeletal maturity indicators to a child's development. This method provided a means with which one can determine the skeletal maturity of a person and thereby determine whether the possibility of potential growth existed.

\section{CONCLUSION}

Since there are individual variations in timing, duration and velocity of growth, skeletal age assessment is essential in formulating viable orthodontic and orthopedic therapy. Bone age determined by hand-wrist radiographs and especially using the TW3 method is suppose to be the most reliable parameter for evaluating skeletal maturation. Skeletal maturity assessed with cervical vertebrae has gained popularity. By using the lateral profiles of the second, third and fourth cervical vertebrae as seen on the routinely taken lateral cephalogram, it is possible to develop a reliable assessment of future adolescent growth potential. To prove the efficacy of this new technique, a study was conducted to evaluate the reliability of cervical vertebral bone age by comparing with the standard hand-wrist bone age by the TW3 method.

\section{CLINICAL SIGNIFICANCE}

This study revealed that the timing and sequence of ossification of the bones in hand and wrist and cervical vertebrae were able to relate the skeletal development of the various skeletal maturity indicators to a child's development. This method provided a means with which one can determine the skeletal maturity of a person and thereby determine whether the possibility of potential growth existed.

\section{REFERENCES}

1. Soegiharto BM, Cunningham SJ, Moles DR. Skeletal maturation in Indonesian and white children assessed with hand-wrist and cervical vertebrae method. Am J Orthod Dentofac Orthop 2008;134:217-226.

2. Hassel B, Farman AG. Skeletal maturation evaluation using cervical vertebrae. Am J Orthod Dentofac Orthop 1995;107:58-66.

3. Green LJ. The interrelationships among height, weight, and chronological, dental and skeletal ages. Angle Orthod 1961;31:189-193.

4. Houston WJB, Miller JC, Tanner JM. Prediction of the timing of the adolescent growth spurt from ossification events in handwrist films. Br J Orthod 1979;6:145-152.

5. Flores-Mir C, Nebbe B, Major PW. Use of skeletal maturation based on hand-wrist radiographic analysis as a predictor of facial growth: a systemic review. Angle Orthod 2004;74:118-124.

6. Fishman LS. Radiographic evaluation of skeletal maturation a clinically oriented method based on hand-wrist films. Angle Orthod 1988;52:89-111. 
7. Grave $\mathrm{KC}$ and Brown T. Carpal radiographs in orthodontic treatment. Am J Orthod Dentofac Orthop 1979;75:27-35.

8. Hagg U, Taranger J. Skeletal ages of the hand and wrist as indicators of the pubertal growth spurt. Acta Odontal Scand 1980;38:187-200.

9. Garcia-Fernandez P, Torre H, Flores L, Rea J. The cervical vertebrae as maturational indicators. J Clin Ortho 1998;32: 270-279.

10. Lamparski GD. Skeletal age assessment utilizing cervical vertebrae. Pittsburgh: Masters thesis, University of Pittsburgh; 1972.

11. Chen F, Terada K, Hanada K. A new method of predicting mandibular length increment on the basis of cervical vertebrae. Angle Orthod 2004;74:630-634.

12. Chertkow $\mathrm{S}$. Tooth mineralization as an indicator of the pubertal growth spurt. Am J Orthod Dentofac Orthop 1980;77:79-91.

13. Mito T, Sato K, Mitani H. Cervical vertebral bone age in girls. Am J Orthod Dentofacial Orthop 2002;122:380-385.

14. Tanner JM, Cameron N, Healy MJR, Goldstein H. Assessment of skeletal maturity and prediction of adult height (TW 3 method). 3rd ed. London: WB Saunders. 2001.p.58-104.

15. Kamal M, Ragini, Goyal S. Comparative evaluation of handwrist radiographs with cervical vertebrae for skeletal maturation in 10-12 years old children. J Indian Soc Pedod Prev Dent 2006;24(3):127-135.

16. Krieg WL. Early facial growth accelerations: a longitudinal study. Angle Orthod 1987;57: 50-62.

\section{ABOUT THE AUTHORS}

\section{CMS Krishna Prasad (Corresponding Author)}

Senior Lecturer, Department of Orthodontics, Army College of Dental Sciences, Secunderabad, Andhra Pradesh, India, e-mail: drcmskp@ gmail.com

\section{Vamsi Nilay Reddy}

Consultant Orthodontist, Chennai, Tamil Nadu, India

\section{Gojja Sreedevi}

Reader, Department of Orthodontics, Kalinga Institute of Dental Sciences, Bhubaneswar, Odisha, India

\section{Swaroopa Rani Ponnada}

Senior Lecturer, Department of Orthodontics, Army College of Dental Sciences, Secunderabad, Andhra Pradesh, India

\section{K Padma Priya}

Senior Lecturer, Department of Orthodontics, MNR Dental College Sangareddy, Andhra Pradesh, India

\section{B Raveendra Naik}

Senior Lecturer, Department of Orthodontics, G Pulla Reddy Dental College, Kurnool, Andhra Pradesh, India 